\title{
Solubility and kinetic properties of deuterium in single crystal $\mathrm{Pd}$
}

\author{
W.C. Chen, Brent J. Heuser* \\ University of Illinois, Department of Nuclear, Plasma, and Radiological Engineering, Urbana, IL 61801, USA
}

Received 18 May 2000; accepted 15 July 2000

\begin{abstract}
The pressure-composition (PC) behavior of deuterium in single crystal Pd has been measured over a temperature range extending from room temperature to $393 \mathrm{~K}$. These data allow the determination of (i) relative partial molar enthalpy and entropy, without the contribution of the configuration entropy, for the dilute solid solution phase, (ii) enthalpy and entropy change characterizing the $\alpha \rightarrow \alpha^{\prime}$ phase transformation, and (iii) Gibbs free energy loss associated with the PC thermodynamic hysteresis. Comparison of these quantities with published data for polycrystal Pd and with our own polycrystal Pd measurements show good agreement, indicating grain boundary interfaces and second phase inclusions known to exist in polycrystalline Pd do not influence these thermodynamic properties. The time dependence of deuterium absorption and desorption from the gas phase has been measured in both single and polycrystal Pd as well. The activation energy and diffusion constant of deuterium in the solid solution phase of Pd agree with published values. The time dependence of deuterium absorption and desorption within the miscibility gap are reduced by approximately two orders of magnitude compared to solid solution behavior. The two-phase kinetic data exhibit an Arrhenius behavior with a factor of two increase in the activation energy compared to diffusion. This is attributed to the phase transformation process controlling the deuterium absorption/desorption kinetic behavior when the Pd-D system is within the miscibility gap. (c) 2000 Elsevier Science B.V. All rights reserved.
\end{abstract}

Keywords: Interstitial alloys; Hydrogen storage materials; Gas-solid reaction; Kinetics; Diffusion

\section{Introduction}

The thermodynamic properties of the solid solution and two-phase regions of metal-hydrogen systems have been theoretically evaluated by Flanagan and co-workers [1,2]. The specific properties of the $\mathrm{Pd}-\mathrm{H}$ and $\mathrm{Pd}-\mathrm{D}$ systems have been experimentally determined for the dilute, solid solution $(\alpha)$ phase in pressure-composition-temperature (PCT) measurements [3-7] and for the $\alpha-\alpha^{\prime}$ two-phase region based on the van't Hoff relationship $[5,8]$. Enthalpy and entropy values in the two-phase region for the $\mathrm{Pd}-\mathrm{H}$ and $\mathrm{Pd}-\mathrm{D}$ systems also have been determined using calorimetric techniques and the van't Hoff relationship, respectively, at 298 [6,9] and $518 \mathrm{~K}$ [10]. The enthalpy and entropy values of Flanagan et al. [9] are in excellent agreement with the results of Lässer and Klatt derived from the van't Hoff relationship [8]. Measurements of the diffusion of hydrogen, deuterium and tritium in the solid solution phase of Pd have been performed over the last several decades $[11,12]$.

To our understanding, all investigations of hydrogen

*Corresponding author. Fax: + 1-217-333-2906.

E-mail address: bheuser@uiuc.edu (B.J. Heuser). behavior in Pd to date have used polycrystal material. The only exception is the work of Labes and McLellan [13], where no obvious effect of grain boundary interfaces on the Sieverts constants for the dilute $\mathrm{Pd}-\mathrm{H}$ solid solution phase is reported in measurements using single crystal $\mathrm{Pd}$ over a temperature range of 475 to $1330 \mathrm{~K}$. The equilibrium thermodynamic properties of deuterium in the solid solution and two-phase regions of single crystal Pd are reported here. The purpose of this study is two-fold. First, PC isothermal measurements of single crystal Pd material were required to establish the correlation between the phase behavior and small-angle neutron scattering measurements [14-16]. The second purpose is to compare the equilibrium thermodynamic properties (enthalpy, entropy, and free energy loss of the pressure hysteresis) of single crystal Pd to that of polycrystal. In addition, kinetic data of deuterium absorption and desorption from the gas phase into and out of single crystal Pd are presented.

\section{Experimental}

Single crystal Pd samples were cut from a 99.999\% pure ingot supplied by Metal Crystals and Oxides of Cam- 
bridge, UK. This ingot was grown by the Czochralski method with [110] cylindrical axis and was approximately $1 \mathrm{~cm}$ in diameter. The polycrystal Pd samples were taken from a $99.9 \%$ pure ingot with similar dimensions supplied by Johnson Matthey Aesar. Typical samples weighed a few grams and had thickness-to-diameter ratios of approximately 0.2 . The sample used for the Sieverts measurement was approximately $6 \mathrm{~g}$ and had an aspect ratio close to one. The extra mass of this sample improved measurement statistics. The surfaces of samples were mechanically polished just before measurement to remove severe surface contamination.

The PCT measurements were performed in an all stainless steel vacuum system with two measurement volumes, 533 and $704 \mathrm{~cm}^{3}$. The isotherms were recorded using small, 15-20 Torr pressure changes to slowly drive each sample across the miscibility gap. The pressure was recorded using MKS Baratron capacitance manometers with full-scale ranges of either 100 or 1000 Torr. The sample temperature was recorded with a K-type thermocouple embedded within the stainless steel mass of the sample holder, but not directly in the gas exposure volume. Experimental trials indicated the measured temperature was within the thermocouple calibration accuracy (between 1 and $2^{\circ}$ ) of the true sample temperature when deuterium gas was present. Only a small portion (approximately $50 \mathrm{~cm}^{3}$ ) of the total gas exposure volume was kept at elevated temperature for measurements above room temperature. The correction to the ideal gas law in this case was insignificant. The temperature of the remaining gas exposure volume was controlled at $305 \mathrm{~K}$. All samples were pre-annealed at $450 \mathrm{~K}$ under a vacuum of $10^{-7}$ Torr for at least $6 \mathrm{~h}$ before the isothermal measurements.

\section{Results and analysis}

\subsection{Equilibrium solubility measurements}

The measured $353 \mathrm{~K}$ isotherms for deuterium in single and polycrystal $\mathrm{Pd}$ are compared to a $353 \mathrm{~K}$ isotherm published by Lässer and Klatt [8] in Fig. 1. Noticeable differences exist between the data sets. In particular, the single crystal absorption and desorption plateau pressure values are shifted downward; 490 Torr (this work) versus 606 Torr (Lässer and Klatt) during absorption and 305 Torr (this work) versus 341 Torr (Lässer and Klatt) during desorption. The single crystal desorption plateau value is in better agreement with a calculated value of 328 Torr using the data of Wicke and Nernst [17]. It is impossible to determine the origin of the different PC behaviors with the limited set of single crystal data. However, the differences are thought to be real, possibly the result of grain boundaries and second phase particles (known to exist in polycrystal Pd [18]) influencing the deuteride formation and reversion processes in the polycrystal material. The

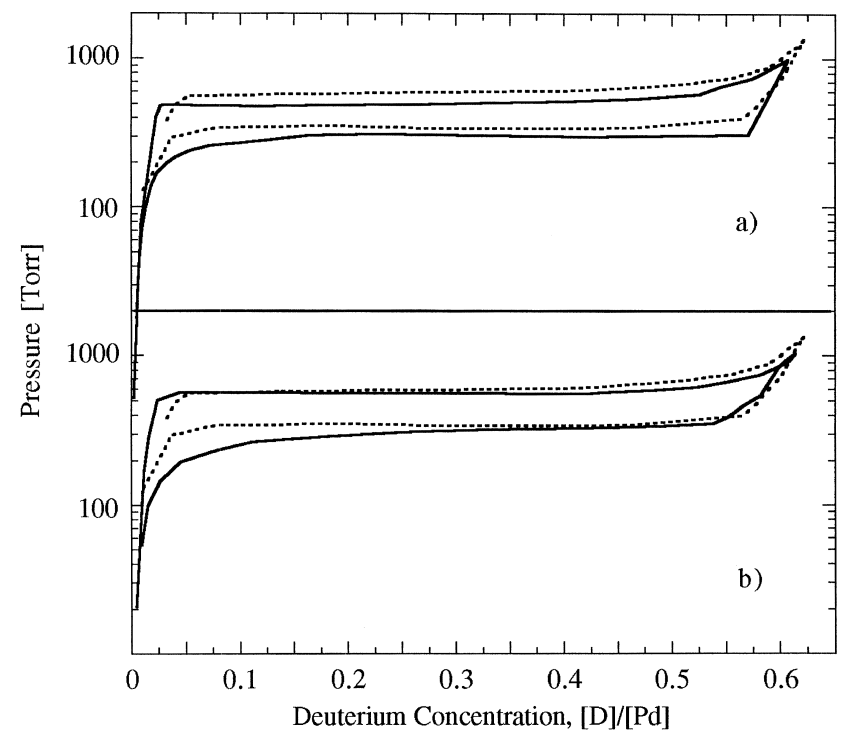

Fig. 1. Measured $353 \mathrm{~K} \mathrm{PC}$ isotherms (solid lines) for (a) single and (b) polycrystal Pd compared to the polycrystal Pd data from Ref. [8] (dotted lines).

polycrystal Pd data are in better agreement with the data of Lässer and Klatt, at least near the boundaries of the miscibility gap.

The PC isothermal data can be used to determine the phase transformation enthalpy and entropy, as well as the free energy loss within the hysteresis loop. A van't Hoff plot, from which the phase transformation enthalpy $\left(\Delta H_{\alpha \rightarrow \alpha^{\prime}}\right)$ and entropy $\left(\Delta S_{\alpha \rightarrow \alpha^{\prime}}\right)$ are determined, for the single and polycrystal Pd solubility data is shown in Fig. 2. The data from Lässer and Klatt [8] are shown for com-

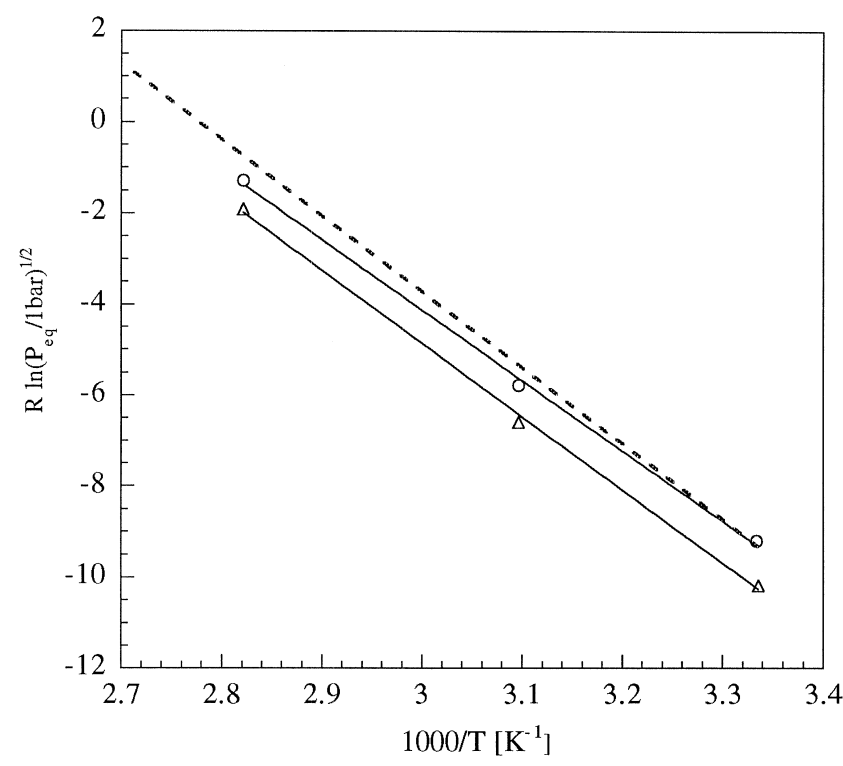

Fig. 2. Van't Hoff plot for single crystal Pd (open triangles), polycrystal Pd (open circles), polycrystal Pd from Ref. [8] (dotted line). The $\alpha \rightarrow \alpha^{\prime}$ phase transformation enthalpy and entropy, listed in Table 1, are obtained from the slope and intercept of the best-fit lines. 
Table 1

Thermodynamic parameters of the solid solution and two-phase regions in $\mathrm{Pd}-\mathrm{D}$

\begin{tabular}{llll}
\hline & $\begin{array}{l}\Delta H_{[\mathrm{D}] \rightarrow 0} \\
\left(\mathrm{~kJ} \mathrm{~mol}^{-1}\right)\end{array}$ & $\begin{array}{l}\Delta S_{[\mathrm{D}] \rightarrow 0}^{\mathrm{E}} \\
\left(\mathrm{J}^{\circ} \mathrm{K}^{-1} \mathrm{~mol}^{-1}\right)\end{array}$ & $\begin{array}{l}\Delta H_{\alpha \rightarrow \alpha^{\prime}} \\
\left(\mathrm{kJ} \mathrm{mol}^{-1}\right)\end{array}$ \\
\hline $\begin{array}{l}\text { Single crystal } \\
\text { this work) }\end{array}$ & $-7.0(2)$ & $-60.8(5)$ & $-16.1(6)$ \\
$\begin{array}{l}\text { Polycrystal } \\
\text { this work) }\end{array}$ & $-7.5(2)$ & $-61.3(5)$ & $-15.5(6)$ \\
$\left(\mathrm{J}_{\alpha \rightarrow \alpha^{\prime}} \mathrm{K}^{-1} \mathrm{~mol}^{-1}\right)$ & $-43(2)$ \\
Ref. [3] & -8.5 & -55.2 & $-42(2)$ \\
Ref. [4] & -7.2 & -58.6 & $-16.8(3)$ \\
Ref. [8] & & & $-46.7(8)$ \\
\hline
\end{tabular}

parison. The phase transformation enthalpy and entropy values derived from Fig. 2 are tabulated in Table 1 and, in general, reasonable agreement is seen.

The free energy loss, $\Delta G_{\text {loss }}$, associated with the pressure hysteresis is given by [1],

$\Delta G_{\mathrm{loss}}=\frac{1}{2} R T \ln \left(\frac{p_{\mathrm{a}}}{p_{\mathrm{d}}}\right)$,

where $p_{\mathrm{a}}$ and $p_{\mathrm{d}}$ are the plateau pressures during absorption and desorption, respectively. The $\mathrm{Pd}-\mathrm{D}$ hysteresis free energy losses calculated from the single crystal and polycrystal PC data and from the isotherms measured by Lässer and Klatt are listed in Table 1.

One explanation of the pressure hysteresis and associated free energy loss in metal-hydrogen systems attributes the effect to dislocation formation during the formation of incoherent phase boundaries. Since the dislocation formation process is irreversible, dislocations must be created during both absorption and desorption to accommodate the volume misfit of the precipitating phase, shifting both pressure plateaus from the true equilibrium behavior. The free energy loss within the hysteresis loop is consistent with the dislocation formation energy, offering indirect, but not conclusive, proof that coherency loss plays this role [19]. The single crystal free energy loss is in moderate disagreement with the two polycrystal $\Delta G_{\text {loss }}$ values in Table 1. This may be an indication that the coherency loss mechanism is altered by the sample microstructure.

The deuterium solubility in the dilute, solid solution phase of Pd is given by Sieverts' law,

$$
\begin{aligned}
\frac{[\mathrm{D}]}{[\mathrm{Pd}]} & =\sqrt{p_{\text {eq }}} \exp \left(-\frac{\Delta H_{[\mathrm{D}] \rightarrow 0}-T \Delta S_{[\mathrm{D}] \rightarrow 0}^{\mathrm{E}}}{R T}\right) \\
& =\sqrt{p_{\text {eq }}} K_{\mathrm{s}}^{-1}
\end{aligned}
$$

where $p_{\text {eq }}$ is the equilibrium $\mathrm{D}_{2}$ gas pressure, $\Delta H_{[\mathrm{D}] \rightarrow 0}$ and $\Delta S_{[\mathrm{D}] \rightarrow 0}^{\mathrm{E}}$ are the relative partial molar enthalpy and entropy (without the contribution of the configurational entropy), respectively, and $K_{\mathrm{s}}$ is the Sievert constant. The solubility parameters for the Pd-D solid solution phase measured in this work and from the published literature (not a complete listing) are given in Table 1. The agreement is reasonable.

\subsection{Absorption/desorption time dependence}

The observed time dependence of the $\mathrm{D}_{2}$ gas pressure change, $p(t)$, during absorption and desorption followed an exponential behavior given by,

$p(t)=p_{0}+\Delta p[1-\exp (-\tau t)]$,

where $p_{0}$ is the starting gas pressure, $p_{\text {eq }}$ is the equilibrium gas pressure, $\Delta p=p_{\mathrm{eq}}-p_{0}$, and $\tau$ is the temperaturedependent time constant. Note that Eq. (3) describes both absorption and desorption, since $p_{\mathrm{eq}}<p_{0}$ for the former and $p_{\text {eq }}>p_{0}$ for the latter. The exponential time constant for cylindrical geometry is related to the diffusion coefficient by,

$\tau=\left[\left(\frac{\pi}{a}\right)^{2}+\left(\frac{2.405}{R}\right)^{2}\right] D$,

where $a$ and $R$ are the sample thickness and radius, respectively, and $D$ is the diffusion coefficient. Eq. (4) is the solution to Fick's second law in cylindrical geometry with a constant surface concentration boundary condition. This is a reasonable boundary condition since the total pressure change during a single absorption or desorption measurement point was small compared to the equilibrium pressure.

The gas pressure time dependence recorded for small, solid solution levels of deuterium in Pd are related to the deuterium diffusion coefficient through Eqs. (3) and (4). Within the miscibility gap, however, diffusion is not the rate limiting step. Instead, the kinetics of deuterium uptake and release are controlled by the phase transformation process. Still, even within the miscibility gap, an exponential time dependence of the gas pressure change and an Arrhenius temperature dependence of the time constant were observed. Accordingly, the diffusion coefficient describing the two-phase behavior is referred to as an effective diffusion coefficient below.

The effective deuterium diffusion coefficients at $353 \mathrm{~K}$ describing deuterium absorption and desorption across the miscibility gap are present in Figs. 3 and 4 for single and polycrystal Pd, respectively. These data have several interesting features. First, the measured deuterium diffusion coefficient in the solid solution $\mathrm{Pd}$ (the maximum solubility of deuterium in $\mathrm{Pd}$ at $353 \mathrm{~K}$ is approximately 


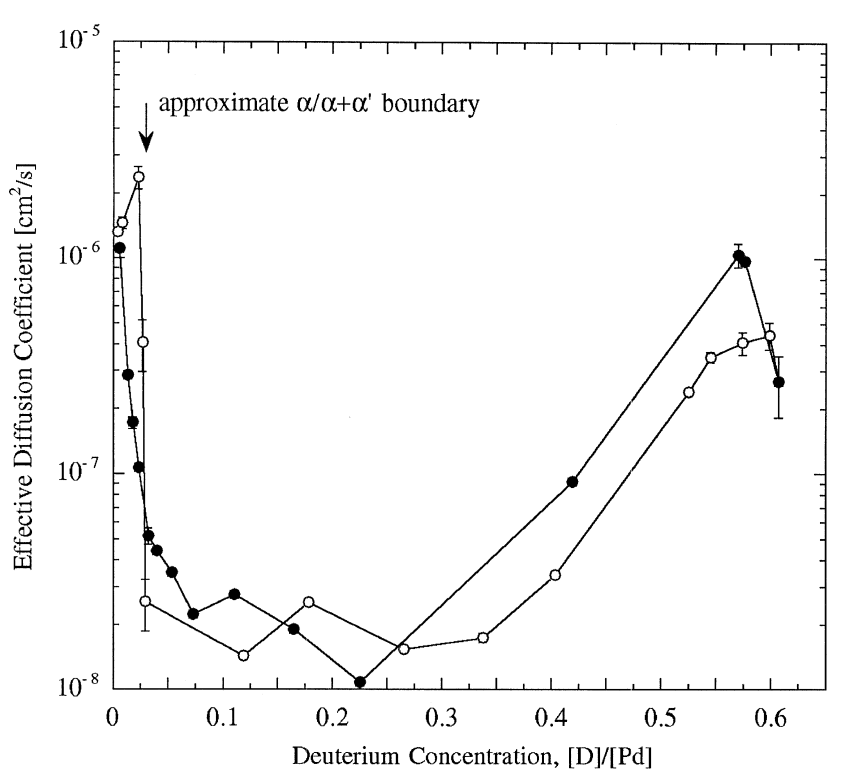

Fig. 3. Effective diffusion coefficient versus deuterium concentration for single crystal Pd during absorption (open circles) and desorption (filled circles) measured at $353 \mathrm{~K}$.

$3 \%$ ) is consistent with the value of $1.6 \times 10^{-6} \mathrm{~cm}^{2} \mathrm{~s}^{-1}$ quoted in Ref. [20]. A significant reduction in the diffusivity is observed as the system enters the miscibility gap. This serves to mark the phase boundary, at least on the low concentration side of the miscibility gap, very well. Notice that the magnitude of the diffusivity and overall trend in both sample types, single and polycrystal, are similar. The diffusion coefficient also approaches the initial value after a complete absorption-desorption cycle. This is proof that

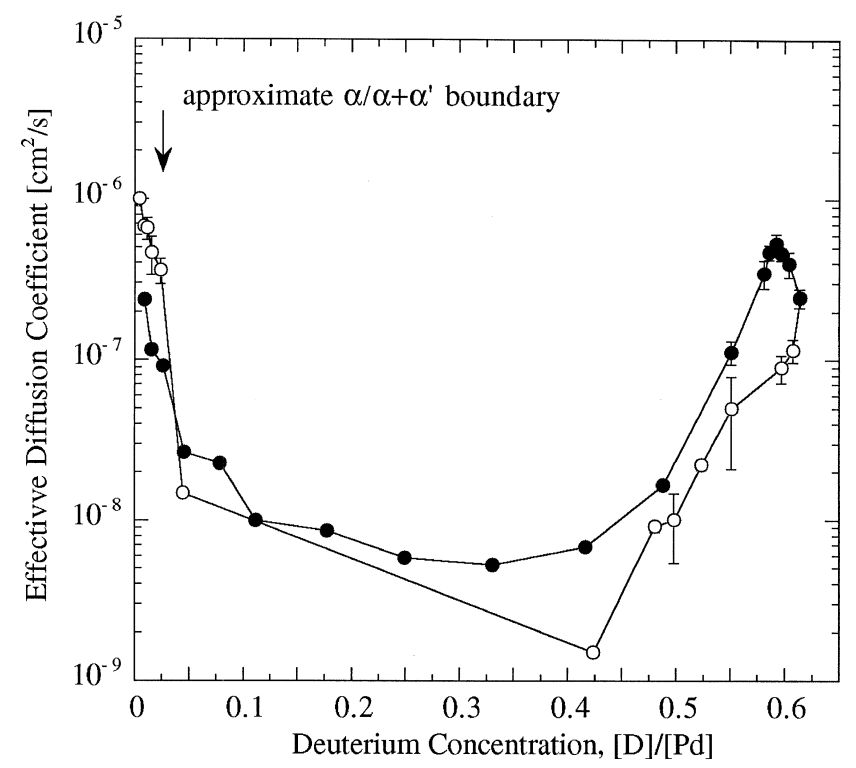

Fig. 4. Effective diffusion coefficient versus deuterium concentration for polycrystal Pd during absorption (open circles) and desorption (filled circles) measured at $353 \mathrm{~K}$.

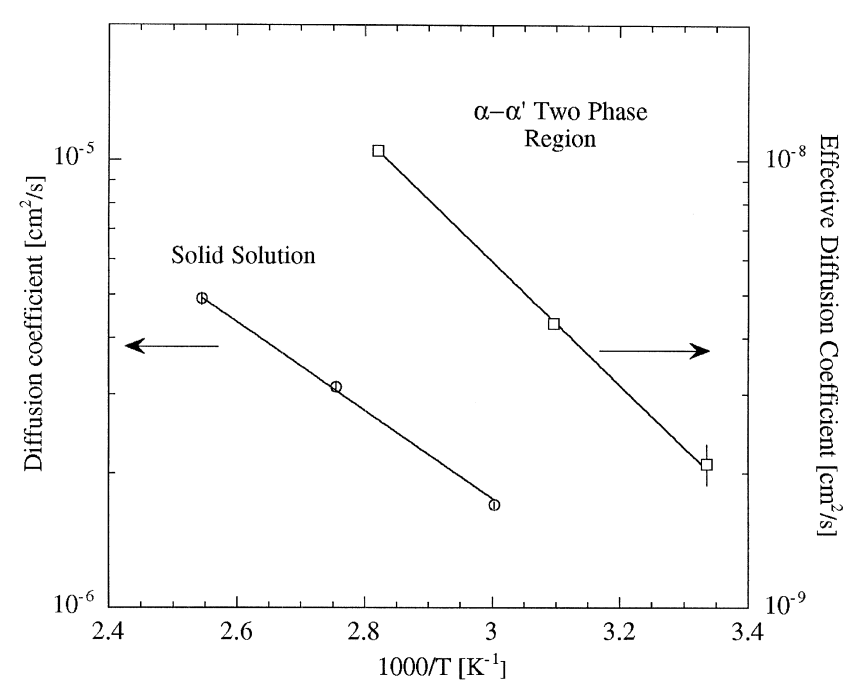

Fig. 5. Arrhenius plot for deuterium in the dilute, solid solution phase (open circles) and in the two-phase region (open boxes) of single crystal Pd.

prolonged gas exposure at $353 \mathrm{~K}$ does not significantly influence the measured time dependence, ruling out the possibility of surface contamination build up during the course of the solubility measurements.

An increase in the effective diffusion coefficient is observed in Figs. 3 and 4 as the miscibility gap is crossed and the system approaches the complete deuteride phase during absorption. Also notice that the effective diffusion coefficient increases immediately upon first desorption for both single and polycrystal material. This reproducible effect corresponds to the downward slope of the PC isotherm as the system approaches the desorption pressure plateau and is coupled to the formation of small, $20 \AA$ thick plates observed during in situ small-angle neutron scattering measurements [21].

The Arrhenius behavior of deuterium diffusion in the solid solution phase and within the two-phase region of single crystal Pd are shown in Fig. 5. Effective diffusion coefficient values below the critical concentration (approximately $0.26[\mathrm{D}] /[\mathrm{Pd}])$ during absorption were used for the $\alpha \rightarrow \alpha^{\prime}$ Arrhenius curve. (Data during desorption were only taken at $353 \mathrm{~K}$ and a curve for this cannot be drawn). The diffusion constant and activation energy for both diffusion and the effective diffusion process within the miscibility gap ( $\alpha \rightarrow \alpha^{\prime}$ transformation) are determined from these data and presented in Table 2.

\section{Conclusions}

1. The equilibrium thermodynamic parameters of the single crystal Pd-D solid solution phase and the $\alpha \rightarrow \alpha^{\prime}$ phase transformation are presented for the first time and are in agreement with previously published values for polycrystal Pd. This indicates these properties are not 
Table 2

Diffusion parameters derived from the time dependence of the $\mathrm{D}_{2}$ gas pressure change

\begin{tabular}{llllll}
\hline & \multicolumn{2}{l}{ Solid solution } & & \multicolumn{2}{l}{ Two-phase region } \\
\cline { 2 - 3 } \cline { 5 - 6 } & $D_{0}\left[\mathrm{~cm}^{2} \mathrm{~s}^{-1}\right]$ & $E_{\mathrm{a}}[\mathrm{eV}]$ & & $D_{0}\left[\mathrm{~cm}^{2} \mathrm{~s}^{-1}\right]$ & $E_{\mathrm{a}}[\mathrm{eV}]$ \\
\hline $\begin{array}{l}\text { Single crystal } \\
\text { (this work) }\end{array}$ & $1.6(3) \times 10^{-3}$ & 0.20 & & $4.6(3) \times 10^{-2}$ & 0.45 \\
\begin{tabular}{l} 
Ref. [20] \\
\hline
\end{tabular} & $1.7 \times 10^{-3}$ & 0.206 & & & \\
\hline
\end{tabular}

influenced by the presence of grain boundaries. This conclusion is expected since the thermodynamic properties investigated are inherent to Pd and should not be affected by a low concentration, interfacial area, or density of lattice defects. Differences have been observed in the $353 \mathrm{~K}$ isothermal behavior of the single crystal material compared to the polycrystalline PC behavior. These differences could be the result of impurity atoms, grain boundary interfaces, and second phase particles known to exist in polycrystalline $\mathrm{Pd}$. Firm conclusions regarding the source(s) of the different PC behavior can not be made with the limited data set presented here.

2. The diffusion constant and activation energy of deuterium in the solid solution phase of single crystal $\mathrm{Pd}$ are both in agreement with published polycrystal Pd values. This again is expected since diffusion should not be influenced by grain boundary interfaces, except perhaps a very low deuterium concentrations or extremely small grain size because of the grain boundarysolute trapping interaction. The effective diffusion coefficient of deuterium within the miscibility gap follows an Arrhenius behavior in single crystal Pd. A factor of two increase in the activation energy is observed in the two phase region (below the critical concentration) compared to deuterium interstitial diffusion in the solid solution phase. This is attributed to the phase transformation process, rather than bulk diffusion, controlling the exchange of deuterium with the gas phase.

3. The free energy loss associated with the pressure hysteresis loop in the single crystal Pd is lower than that of polycrystalline Pd. This, together with the noted differences in PC behavior, could be an indication that the phase transformation process is altered by the lack of grain boundary interfaces and second phase particles in the single crystal material.

\section{Acknowledgements}

Acknowledgment is made to the donors of The Petroleum Research Fund, administered by the American Chemical Society, for support of this research. The authors also express their gratitude to Dr. T. Flanagan (Univ. Vermont) for useful comments regarding the manuscript.

\section{References}

[1] T.B. Flanagan, C.-N. Park, W.A. Oates, Progr. Solid State Chem. 23 (1995) 291.

[2] T.B. Flanagan, W.A. Oates, Ber. Bunsenges. Phys. Chem. 76 (1972) 706.

[3] J.D. Clewley, T. Curran, T.B. Flanagan, W.A. Oates, J. Chem. Soc., Faraday Trans. I 69 (1973) 449.

[4] E. Fromm, H. Jehn, Bull. Alloy Phase Diagr. 5 (1984) 324.

[5] R. Lässer, Tritium and Helium-3 in Metals, Springer, Berlin, 1989.

[6] T. Kuji, W.A. Oates, B.S. Bowerman, T.B. Flanagan, J. Phys. F: Met. Phys. 13 (1983) 1785.

[7] H. Katsuta, R.B. McLellan, Scripta Metall. 12 (1978) 1161.

[8] R. Lässer, K.H. Klatt, Phys. Rev. B 28 (1983) 748.

[9] T.B. Flanagan, W. Luo, J.D. Clewley, J. Less-Common Metals 172-174 (1991) 42

[10] C. Picard, O. Kleppa, G. Boureau, J. Chem. Phys. 69 (1978) 5549.

[11] J.Völkl, G. Alefeld, in: A.S. Nowick, J.J. Burton (Eds.), Diffusion in Solids, Academic Press, New York, 1975.

[12] Y. Fukai, in: U. Gonser (Ed.), The Metal-Hydrogen System, Springer, Berlin, 1993

[13] C. Labes, R.B. McLellan, Acta Metall. 26 (1978) 893-899.

[14] B.J. Heuser, J.S. King, W.C. Chen, J. Alloys Comp. 292 (1999) 134.

[15] B.J. Heuser, J.S. King, W.C. Chen, J. Alloys Comp. 305 (2000) 318.

[16] W.C. Chen, B.J. Heuser, J.S. King, J. Appl. Crystallogr. 33 (2000) 442.

[17] E. Wicke, G. Nernst, Ber. Bunsenges. Physik. Chem. 68 (1964) 224.

[18] B.J. Heuser, J.S. King, G.C. Summerfield, Defect-Interface Interactions, Mat. Res. Soc. Symp. Proc. Vol. 319, 1994, p. 339.

[19] T.B. Flanagan, B.S. Bowerman, G.E. Biehl, Scripta Metall. 14 (1980) 443

[20] J. Völk1, G. Wollenweber, K.-H. Klatt, G. Alefeld, Z. Naturforch (a) 26 (1971) 922.

[21] W.C. Chen, B.J. Heuser, Phys. Rev. Lett., submitted. 